\title{
Clinical Feasibility of Zero TE Skull MRI in Patients with Head Trauma in Comparison with CT: A Single-Center Study
}

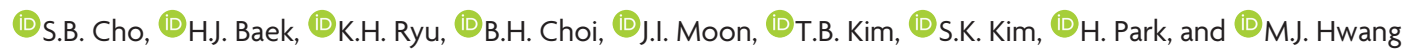

\begin{abstract}
BACKGROUND AND PURPOSE: Conventional MR imaging techniques cannot produce optimal images of bone structures because bone has little water and a very short T2 life span. The aim of this study was to investigate the clinical feasibility of skull MR imaging using the zero TE sequence in patients with head trauma by assessing its diagnostic image quality and quantitative measurement compared with CT images.
\end{abstract}

\begin{abstract}
MATERIALS AND METHODS: Thirteen enrolled patients with head trauma were assessed using brain CT and skull MR imaging. Image quality was graded on a 5-point Likert scale to compare the 2 modalities. To evaluate quantitative analyses between the 2 imaging modalities, we measured skull thickness and normalized bone tissue signal. Interobserver reliability was assessed using weighted $\kappa$ statistics and the intraclass correlation coefficient.

RESULTS: Both imaging techniques clearly depicted skull fractures in all 13 patients. The mean scores for skull MR imaging and CT were $4.65 \pm 0.56$ and $4.73 \pm 0.45(P=.157)$, respectively, with substantial interobserver agreement $(P<.05)$. The 2 imaging modalities showed no difference in skull thickness $(P=.092)$ and had good correlation $\left(r^{2}=0.997\right)$. The mean value of normalized bone tissue signal among the 3 layers of the skull was relatively consistent $(P=.401)$ with high interobserver agreement $(P<.001)$.
\end{abstract}

CONCLUSIONS: Zero TE skull MR imaging has diagnostic image quality comparable with that of CT images. It also provides consistent results on the quantitative measurement of cortical bone with $\mathrm{CT}$ images.

ABBREVIATION: ZTE $=$ zero TE

M $\mathrm{R}$ imaging is a noninvasive technique that obtains excellent soft-tissue contrast and high resolution of anatomic detail in the body without radiation. ${ }^{1}$ However, it is unsuitable for depicting cortical bone structures because of low proton density (approximately 20\% water) and a very short $\mathrm{T} 2$ relaxation time (approximately $390 \mu$ s at 3T). ${ }^{2}$ By contrast, CT is the most optimal technique for revealing bone structures in images with high spatial resolution, fast acquisition, and high availability; however, its capability in imaging soft tissue is poor, and radiation exposure is a major drawback. ${ }^{3-8}$ In clinical practice, MR imaging is an essential diagnostic technique because of its inherent advantage in

Received July 30, 2018; accepted after revision November 1.

From the Departments of Radiology (S.B.C., H.J.B., K.H.R., B.H.C., J.I.M., T.B.K.) and Neurosurgery (S.K.K., H.P.), Gyeongsang National University Changwon Hospital, Gyeongsang National University School of Medicine, Changwon, Republic of Korea; and MR Applications and Workflow (M.J.H.), GE Healthcare Korea, Seoul, Republic of Korea.

Please address correspondence to Hye Jin Baek, MD, PhD, Department of Radiology, Gyeongsang National University Changwon Hospital, Gyeongsang National University School of Medicine, 11 Samjeongja-ro, Seongsan-gu, Changwon 51472 Republic of Korea; e-mail: sartre81@gmail.com

http://dx.doi.org/10.3174/ajnr.A5916 establishing a diagnosis and treatment plan for various intracranial diseases. Therefore, MR imaging would be the most ideal diagnostic imaging technique if it could provide reliable clinical information about bone structures and soft tissues. There is an increasing clinical need to resolve the limitations of MR imaging with regard to bone structures. MR bone imaging is increasingly becoming a focus of interest in the field of musculoskeletal radiology, MR imaging-based attenuation correction in PET, MR imaging-based radiation therapy planning, and MR imagingguided focused sonography. ${ }^{9}$

Concerning the technical aspects of conventional MR imaging, optimal images of bone structures cannot be obtained because the minimum TE for the spin-echo and gradient-echo pulse sequences is too slow (approximately $8-10$ and $1-2 \mathrm{~ms}$, respectively) to detect a meaningful bone signal. ${ }^{2}$ Compared with conventional sequences, the ultrashort TE sequence with a center-out $k$-space acquisition can allow sufficiently fast data acquisition of the rapidly decaying bone signal. ${ }^{10,11}$ Long T2-suppression methods (eg, echo subtraction, saturation prepulses, or multiple sequences) are generally applied to separate bone from soft tissue, to 
achieve selective bone images using an ultrashort TE sequence. ${ }^{12}$ The sequence for MR bone imaging was recently developed to visualize very short $\mathrm{T} 2$ relaxation of the object, based on $3 \mathrm{D}$ radial zero TE (ZTE), and it can provide high-resolution isotropic images with fast and silent scanning. ${ }^{13-15}$ To date, MR bone imaging using ultrashort TE or ZTE sequences has been studied for $\mathrm{PET} / \mathrm{MR}$ imaging attenuation correction from the perspective of the technical approach in the literature. ${ }^{9,16-20}$

Recently, ZTE bone MR imaging was applied to osseous shoulder imaging, which showed strong intermodality agreement between measurements and grades of the lesions from ZTE bone MR imaging and CT. ${ }^{21}$ However, to the best of our knowledge, there is no study of the clinical application of MR bone imaging of the skull for diagnostic use. Therefore, this study aimed to investigate the clinical feasibility of using ZTE skull MR imaging for evaluating skull lesions in patients with head trauma by assessing its diagnostic image quality and quantitative measurement compared with CT images.

\section{MATERIALS AND METHODS Patient Characteristics}

A review of the data base at Gyeongsang National University Changwon Hospital identified patients with head trauma who were admitted to the emergency department and underwent routine brain CT for the evaluation of intracranial or skull abnormalities between June 2017 and May 2018. Using electronic medical records and a PACS, we selected 16 patients who also had undergone ZTE skull MR imaging as the follow-up imaging. We ultimately enrolled 13 of 16 patients. Three patients were excluded because of poor image quality from uncontrolled motion artifacts (CT: $n=2$; MR imaging: $n=1$ ). The 13 patients who were included in this study comprised 4 women and 9 men with a mean age of $43.8 \pm 12.7$ years (range, $24-68$ years). The average interval between the initial CT and ZTE skull MR imaging examinations was $10.1 \pm 7.4$ days (range, $1-23$ days).

In the present study, all retrospective data collection and analyses were conducted in accordance with our local institutional review board guidelines, after obtaining its approval. The institutional review board determined that patient approval and informed consent were not required for retrospective review of images and electrical medical records. The patients' records and information were anonymized and de-identified before analysis.

\section{ZTE Skull MR Imaging}

In general, ZTE uses a nonselective radiofrequency excitation pulse and a $3 \mathrm{D}$ radial center-out $k$-space trajectory. The readout gradients are ramped up before the radiofrequency excitation (Fig $1 A)$. The pulse is grouped into segments, and each segment contains numerous spokes. These gradients are changed slightly and are not ramped down between spokes. The minimal gradient switching between repetitions minimizes eddy currents to a negligible level and reduces acoustic noise level. ${ }^{22}$ The imaging encoding starts immediately after the end of the radiofrequency excitation to fill the center of the $k$-space, which is the nominal ZTE. The radiofrequency duration must remain short (approximately $8-16 \mu \mathrm{s}$ ), and the flip angle is limited in ZTE to minimize the delay between the radiofrequency pulse and the transmit-to-re-
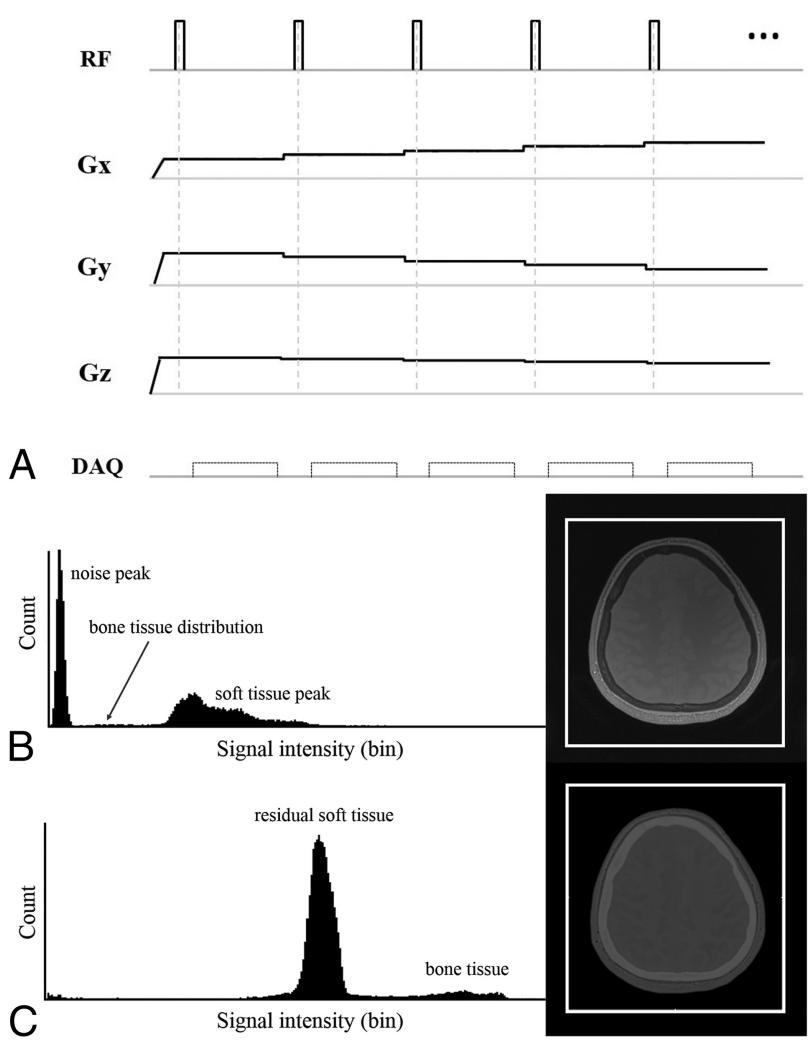

FIG 1. A, ZTE pulse sequence diagram of a segment with 5 spokes (the actual scan contains 384 spokes per segment). The ZTE sequence uses a hard radiofrequency (RF) pulse and switch data acquisition (DAQ) just after the RF to receive the free induction decay signal, which results in a nominal ZTE. The $k$-space fills the 3D radial center. The readout gradients (Gx, Gy, Gz) are ramped up before the RF and change in small steps. Such a small gradient change results in a fast, silent scan. $B$ and $C$, Histogram-based intensity-correction has been used to generate the CT-like bone image. There are 2 typical intensity histograms and corresponding axial images. $B$, The ZTE dataset of proton-density contrast. C, The final dataset of the CT-like contrast image with further postprocessing. After we applied inversion logarithmic image rescaling, the CT-like contrast image reveals excellent cortical bone delineation. On each histogram, the horizontal axis represents the signal intensity and the vertical axis represents the count.

ceive switching time. In addition, to prevent disturbing the spin excitation substantially, the excitation bandwidth is limited. As a result, the contrast is a native proton-density contrast.

After acquiring the proton-density image using ZTE, we applied a bias-correction algorithm to neaten the soft-tissue signal intensities and correct signal inhomogeneity owing to coil geometry and variable tissue cross-sections. ${ }^{9,20,21}$ The histogram distribution of the inverse log-scaled images was then used for the CT-like contrast images to remove the background noise while retaining bone and soft tissue (Fig $1 B,-C) .^{9,20,21}$ The histogram of the proton-density image easily yields 2 groups: 1) soft tissues such as white matter, gray matter, CSF, muscle, and fat; and 2) background air. Then, the median of the tissue signals, which are magnified to the proper level, is used to determine the threshold values. The imaging value, which is higher than the bone threshold, is magnified and generates a CT-like contrast image.

\section{ZTE Skull MR Imaging Scan Parameters}

MR imaging was performed using a 3T system (Signa Architect; GE Healthcare, Milwaukee, Wisconsin) with a 48-channel head 
coil. The ZTE proton-density image was acquired in the axial plane to cover the whole brain in 4 minutes 52 seconds using the following parameters: TE, $0.016 \mathrm{~ms}$ (nominal TE $=0$ ); TR, 2.65 $\mathrm{ms}$; FOV, $240 \times 240 \mathrm{~mm}$; slice thickness, $2 \mathrm{~mm}$; flip angle, $1^{\circ}$; spokes per segment, 384 ; matrix size, $288 \times 288$; image voxel resolution, $0.8 \times 0.8 \times 1 \mathrm{~mm}^{3}$; receiver bandwidth, $\pm 31.25 \mathrm{kHz}$; and total number of scans, 3 .

\section{Brain CT Scan Parameters}

Skull CT images were obtained using the routine brain protocol on 2 different CT machines with the following acquisition parameters: 1) IQon Spectral CT (Philips Healthcare, Best, the Netherlands) $-120 \mathrm{kV}$ (peak); $200 \mathrm{mAs}$; collimation, $16 \times 0.625 \mathrm{~mm}$; pitch factor, 0.985; rotation time, 0.33 seconds; FOV, $250 \mathrm{~mm}$; slice thickness, $3 \mathrm{~mm}$; and slice increment, $0.4 \mathrm{~mm}$; and 2) Aquilion ONE/ViSION Edition CT (Toshiba Medical Systems, Tokyo, Japan)—120 kVp; $190 \mathrm{mAs}$; collimation, $80 \times 0.5 \mathrm{~mm}$; pitch factor, 0.985; rotation time, 0.75 seconds; FOV, $240 \mathrm{~mm}$; slice thickness, $3 \mathrm{~mm}$; and slice increment, $0.4 \mathrm{~mm}$.

\section{Image Analyses}

All datasets were anonymized with randomization. Two readers reviewed all images using the PACS. Two attending neuroradiologists with 11 and 8 years of experience independently analyzed all ZTE skull MR images and CT scans to evaluate the image quality of skull MR imaging from a clinical feasibility perspective. They also evaluated whether a skull fracture was present in both image sets. In the review of all images, the window width and window level could be modified for evaluation. The 2 types of skull images were assessed separately to minimize bias because of the results of the other images. For the initial interpretation, each reader was provided the ZTE skull MR images, and they analyzed all images twice with an interval of 2 weeks between each analysis. After 2 weeks, the readers were provided the CT images of the enrolled patients. They analyzed all these images twice, using the same interval as in ZTE skull MR imaging interpretation. Image-quality measures for each image set were evaluated for following items: 1 ) the conspicuity and differentiation of 3 layers of skull structures (ie, outer table, diploic layer, and inner table), 2) clear visualization of the suture lines, 3) visualization and demarcation of the fracture line, and 4) the presence of artifacts. The readers then scored image quality on the following 5-point Likert scale: 1) nondiagnostic (ie, not acceptable for diagnostic use); 2) unacceptable (ie, not acceptable for diagnostic use); 3) sufficient (ie, acceptable for diagnostic use but with minor issues); 4) diagnostic (ie, acceptable for diagnostic use); and 5) excellent (ie, acceptable for diagnostic use).

To evaluate the geometric concordance of skull structures on the ZTE skull MR imaging and CT images, the readers measured skull thickness manually from the most inner cortex to the most outer cortex in 6 regions from each corresponding slice of the same patient on the MR imaging and CT images. For each patient, 3 slices were selected to measure skull thickness in both frontal, both parietal, both temporal, and both occipital bones.

The same readers also measured the signal intensities and Hounsfield units of the outer table, diploic layer, and inner table on the ZTE skull MR imaging and CT images. The readers defined the ROI as $15 \mathrm{~mm}^{3}$ symmetrically in the outer cortex, diploic space, and inner cortex from each corresponding slice of the same patient on the MR imaging and CT images. The same neuroradiologists manually drew 6 ROIs in both frontal, both parietal, and both occipital bones from each patient on 3 selected slices on the MR imaging and CT images. To normalize the signal intensity of ZTE skull MR imaging, we calculated the normalized signal intensity of each skull layer by dividing its signal intensity by that of the background region and then multiplied this value by 100 . We calculated the ratio of bone signal intensity of ZTE skull MR imaging to the bone density of CT images and termed this value the "normalized bone tissue signal." After the readers' independent analyses, the same neuroradiologists conducted image analysis in consensus to make a reference standard for deciding the presence of skull fracture and its location.

\section{Statistical Analysis}

The data were tested for normal distribution using the Kolmogorov-Smirnov test. Continuous variables were expressed as mean $\pm \mathrm{SD}$. The image-quality assessments of ZTE skull MR imaging and CT were assigned numeric values. The mean values of the readers' ratings were not directly statistically compared because these values were not strictly continuous variables. However, we decided to present a summary of the readers' ratings for ZTE skull MR imaging and CT, which are expressed as mean \pm $\mathrm{SD}$. The scores of each image set from the 2 readers were averaged, and the Wilcoxon signed rank test was used to compare the scores of the ZTE skull MR imaging and CT images. Interobserver agreement between 2 readers was calculated by weighted $\kappa$ statistics. On the basis of the description by Landis and Koch, ${ }^{23}$ the $\kappa$ value was interpreted as follows: 0 , no agreement; $0-0.19$, poor agreement; $0.20-0.39$, fair agreement; $0.40-0.59$, moderate agreement; $0.60-0.79$, substantial agreement; and $0.80-1.00$, nearly perfect agreement. The skull thickness was compared and correlated between ZTE skull MR imaging and CT images using the Wilcoxon signed rank test and Spearman correlation coefficient analysis after the values of each image set from the 2 readers were averaged. The Kruskal-Wallis test was used to evaluate differences in the normalized bone tissue signal among the 3 layers of the skull. The interobserver agreement between the 2 readers was also assessed with the intraclass correlation coefficient. All statistical analyses were conducted using SPSS, Version 24.0 (IBM, Armonk, New York). A $P$ value $<.05$ was statistically significant.

\section{RESULTS}

Skull images were successfully obtained from all patients using the ZTE technique. Skull structures were clearly depicted on the ZTE skull MR images and matched well with those depicted on the CT images. Two attending neuroradiologists independently reviewed all acquired ZTE skull MR imaging and CT images without difficulty in detecting the fracture sites. All 13 patients had a skull fracture (Figs 2 and 3). In addition, the skull suture lines were conspicuously demarcated on the ZTE skull MR imaging in all 13 patients.

Patient data and the scores of the overall image quality for ZTE skull MR imaging and CT images determined by the 2 readers are presented in the Table. The mean scores of ZTE skull MR imaging 


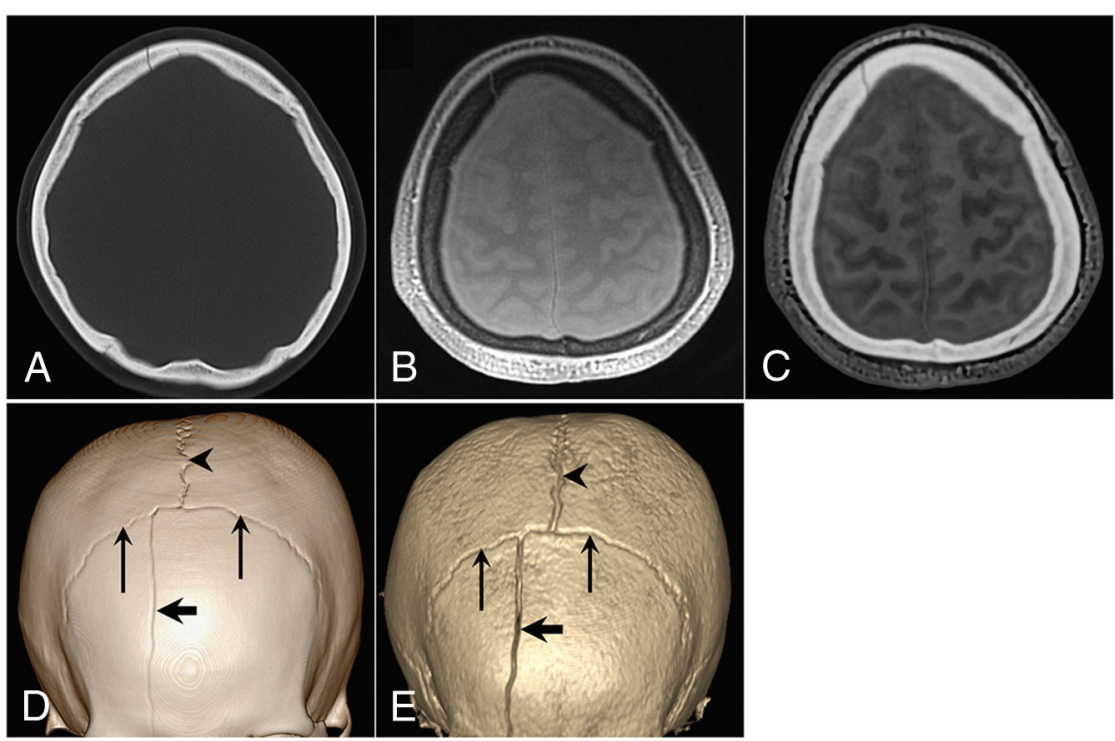

FIG 2. A 42-year-old woman with a right frontal bone fracture. A, Axial CT image. B, Axial proton-density ZTE image. C, Axial CT-like contrast ZTE image. 3D volume-rendered CT image (D) and ZTE skull MR imaging (E). A linear skull fracture line is visible in the right frontal bone on the CT and MR images (thick arrows). The coronal sutures (thin arrows) and sagittal sutures (arrowheads) are depicted.

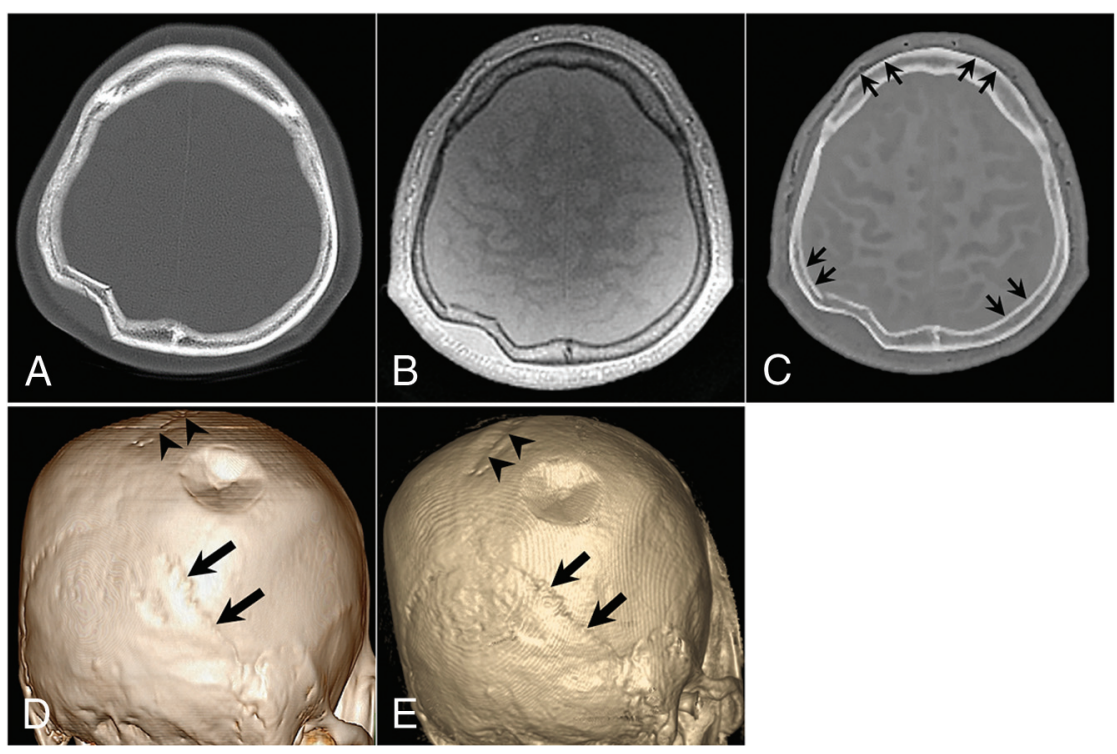

FIG 3. A 52-year-old man with a right parietal bone fracture. $A$, Axial CT image. $B$, Axial protondensity ZTE image. C, Axial CT-like contrast ZTE image. 3D volume-rendered CT image $(D)$ and ZTE skull MR imaging $(E)$. A focal depression fracture is visible in the right parietal bone. The sagittal suture (arrowheads) and bilateral lambdoidal sutures (thick arrows) show conspicuous delineation. Subtle marginal artifacts exist along the inner cortex of both parietal bones and the outer cortices of both frontal bones. The artifacts have a short segmental stepped appearance, which may be related to the postprocessing of histogram-based intensity correction (short thin arrows) in C.

was not statistically different: $9.5 \pm 4.3$ and $9.5 \pm 4.2 \mathrm{~mm}$, respectively $(P=$ .092). Skull thickness measured on the ZTE skull MR imaging and CT images also showed good correlation $\left(r^{2}=\right.$ 0.997, $P<.001)$. Based on the results of the intraclass correlation coefficient, interobserver agreement of the skull thickness was as follows: CT, 0.989 (95\% CI, $0.984-0.992 ; P<.001)$ and MR imaging, 0.977 (95\% CI, 0.967-0.985; $P<$ .001). The mean value of the normalized bone tissue signal tended to be higher in the diploic space than in the outer and inner cortices $(0.45 \pm 0.13$ versus $0.43 \pm$ 0.11 versus $0.41 \pm 0.01$, respectively); however, there was no statistical difference among the 3 layers of the skull $(P=$ $.401)$ (Fig. 4). For interobserver agreement, the intraclass correlation coefficient for the normalized bone tissue signal was 0.885 (95\% CI, 0.851-0.911; $P<.001)$.

\section{DISCUSSION}

The findings of our study indicated that ZTE skull MR imaging was suitable for identifying bone structures in the skull. Its diagnostic image quality was comparable with that of CT images for evaluating traumatic skull lesions. In addition, this study provided a quantitative evaluation of ZTE skull MR images by a direct comparison with CT images and showed good correlation between the 2 skull images.

To date, MR bone imaging is challenging because the magnetization from hydrogen atoms in the cortical bone demonstrates much faster transversal relaxation than other body tissues, and the available magnetization is relatively low because of decreased proton density. ${ }^{24}$ This phenomenon is caused by the characteristics of bone tissue, which has little water and a very short T2 life span. ${ }^{2}$ With technical advances, MR bone imaging has proved technically

and CT images were $>4$ points with acceptable image quality for diagnostic use. For both readers, the mean score of ZTE skull MR was slightly lower than that of CT images. However, the average mean scores for skull MR imaging and brain CT images by the readers were not statistically significant $(4.65 \pm 0.56$ versus $4.73 \pm 0.45, P=.157)$. Substantial interobserver agreement was observed for the overall image quality of skull MR imaging $(\kappa=$ $0.829, P=.001)$ and brain CT $(\kappa=0.806, P=.003)$.

The skull thickness on ZTE skull MR imaging and CT images feasible using ultrashort TE and ZTE sequences in previous studies. ${ }^{10-15}$ In contrast to ultrashort TE sequences, MR bone imaging using the ZTE sequence has a greater signal-to-noise ratio with scan time efficiencies and it provides isotropic high-resolution images with multiplanar reconstruction. ${ }^{13-15,21}$ To date, a few subsequent studies have focused on MR bone imaging in the field of PET. ${ }^{2,9,11,16-20}$ However, MR bone imaging has not been studied from a diagnostic perspective in the field of neuroradiology, though interest in this topic is increasing. 


\begin{tabular}{|c|c|c|c|c|c|c|c|c|}
\hline \multirow[b]{2}{*}{ Patient No. } & \multirow[b]{2}{*}{ Age (yr) } & \multirow[b]{2}{*}{ Sex } & \multirow[b]{2}{*}{ Fracture Location } & \multirow{2}{*}{$\begin{array}{l}\text { Interval between ZTE } \\
\text { Skull MRI and CT }\end{array}$} & \multicolumn{2}{|c|}{ ZTE Skull MRI } & \multicolumn{2}{|c|}{ Brain CT } \\
\hline & & & & & Reader 1 & Reader 2 & Reader 1 & Reader 2 \\
\hline 1 & 52 & $M$ & Right parietal & 20 Days & 5 & 5 & 5 & 5 \\
\hline 2 & 42 & $\mathrm{~F}$ & Right frontal & 17 Days & 5 & 5 & 5 & 5 \\
\hline 3 & 47 & M & Right temporal & 8 Days & 5 & 5 & 5 & 5 \\
\hline 4 & 68 & M & Right occipital & 23 Days & 4 & 4 & 4 & 5 \\
\hline 5 & 25 & $\mathrm{~F}$ & Right temporal & 2 Days & 3 & 4 & 4 & 4 \\
\hline 6 & 24 & M & Left occipital & 3 Days & 5 & 5 & 5 & 5 \\
\hline 7 & 38 & M & Right frontal & 11 Days & 4 & 4 & 4 & 4 \\
\hline 8 & 41 & M & Left temporal & 1 Day & 5 & 5 & 5 & 5 \\
\hline 9 & 57 & M & Left parietal & 7 Days & 5 & 5 & 5 & 5 \\
\hline 10 & 53 & M & Right frontal & 14 Days & 4 & 4 & 4 & 4 \\
\hline 11 & 52 & $\mathrm{~F}$ & Right temporal & 16 Days & 5 & 5 & 5 & 5 \\
\hline 12 & 31 & M & Right temporal & 2 Days & 5 & 5 & 5 & 5 \\
\hline 13 & 41 & $\mathrm{~F}$ & Right parietal & 7 Days & 5 & 5 & 5 & 5 \\
\hline
\end{tabular}

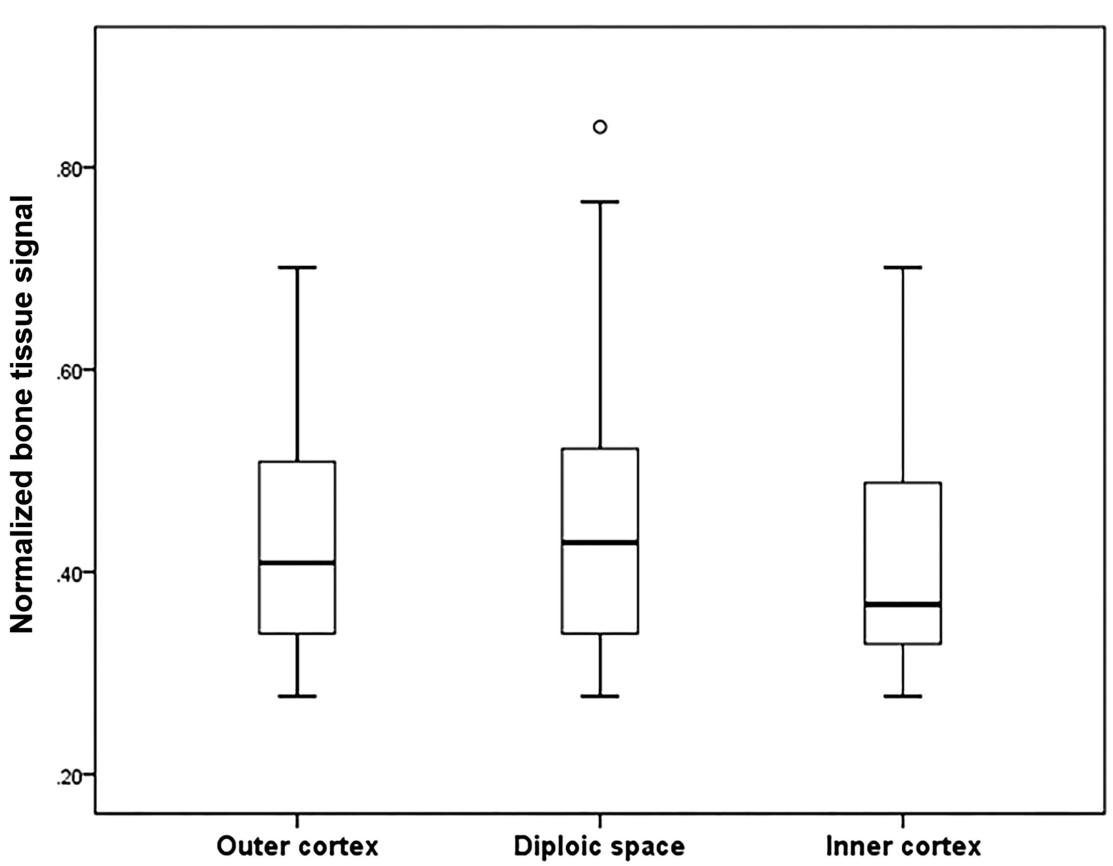

FIG 4. Boxplot of the normalized bone tissue signal of the outer cortex, diploic space, and inner cortex. The line across the box represents the median value. The box ends represent the first and third quartiles. The end points in each graph represent the smallest and largest values. The median ratio of the normalized bone tissue signal is highest in the diploic space of the skull; however, there is no significant difference among the 3 skull layers.

In the present study, we applied the ZTE sequence to obtain images of the skulls of patients with head trauma. Our findings showed results similar to those of previous studies from the perspective of its clinical feasibility in assessing the head. ${ }^{9,19,20}$ For all 13 patients, ZTE skull MR imaging was successfully acquired, and skull suture lines with skull fractures were depicted as clearly on the ZTE skull MR imaging as on the CT images. Our results for detecting bone abnormalities were similar to those of a recent study, ${ }^{21}$ which showed comparable agreement for evaluating osseous lesions in the shoulder between raters and modalities. In the present study, the CT-like image with positive contrast for the skull obtained by postprocessing allowed more intuitive interpretation for evaluating the skull, and it is also consistent with the previous study, despite differences in the applied anatomic sites.

We also assessed the overall imaging quality of skull MR imaging, and ours is the first study to evaluate the image quality of skull MR imaging for diagnostic use. ZTE skull MR imaging yielded acceptable image quality of $>4$ points with substantial interobserver agreement in the present study. There was no significant difference, though the mean score of ZTE skull MR images was slightly lower than that of CT images because moderate motion artifacts of a few slices occurred in 1 patient owing to the patient's irritability, which was closely associated with the longer scan time of ZTE skull MR imaging than CT. For clinical use, further technologic effort to reduce the scan time is essential to expand the indications of ZTE skull MR imaging for various clinical situations.

We were also able to identify subtle marginal irregularities on the only CTlike images of ZTE skull MR imaging (2/13 patients, $15.4 \%)$, though there were no demonstrable motion artifacts in the 2 patients. These artifacts had a thin, short segmental stepped appearance in the inner or outer cortex of both frontal and parietal bones (Fig 3C); however, these artifacts did not have a crucial impact for diagnosis by the 2 readers. The reason for this finding is unclear, but it may be related to the postprocessing of histogram-based intensity correction to separate bone tissue from other tissue. We expect that this issue of marginal stepped artifacts can be solved if the algorithm for the postprocessing of ZTE skull MR imaging is improved.

In the current study, the 2 imaging modalities showed no statistically significant differences in the skull thickness measurement, and they had good correlation in skull thickness measurements. In contrast to the finding of a previous study, ${ }^{21}$ we measured the normalized bone tissue signal and found that the normalized bone tissue signal from each skull layer was relatively consistent on the basis of a direct comparison of the signal-tonoise ratio on ZTE skull MR imaging and Hounsfield units on CT for the same regions. In addition, the skull-thickness measure- 
ments and the normalized bone tissue signal showed high interobserver agreement. The mean value of the normalized bone tissue signal was higher in the diploic space than in the other skull layers. This result could reflect the characteristics of the tissue in the diploic space, which has a fat component in the marrow cavity; this factor results in a short T1 relaxation time in MR imaging and a decreased number of Hounsfield units on the CT image. These quantitative comparison results of the 2 imaging modalities imply that ZTE skull MR imaging could reproduce images close to the skull itself and suggest that ZTE skull MR imaging could be a valid alternative to CT for skull imaging in a variety of clinical situations. Furthermore, these results also support the findings of previous studies ${ }^{9,20}$ that suggested possible technical applications to develop attenuation correction algorithms.

With regard to the technical aspect, hard pulse sequences such as ZTE require a higher readout bandwidth to allow shorter encoding times and less $\mathrm{T} 2{ }^{\star}$ blurring. ${ }^{9,25}$ The flip angle below the corresponding Ernst angle is also important to obtain native proton-density-weighted images and achieve appropriate tissue differentiation during the postprocessing. ${ }^{9}$ In a previous study, ${ }^{9}$ the authors performed ZTE skull MR imaging based on the default high-resolution protocols using different imaging bandwidths $( \pm 31.25 \mathrm{kHz}, \pm 62.5 \mathrm{kHz}$, and $\pm 125 \mathrm{kHz})$ and flip angles $\left(0.6^{\circ}\right.$, $1.2^{\circ}$, and $2.4^{\circ}$ ). They found that images with the highest bandwidth and lowest flip angle were sharpest and had the least blurring at the tissue interfaces, whereas the images with the lowest bandwidth and highest flip angle had the highest signal-to-noise ratio and soft-tissue contrast with partial $\mathrm{T} 1$ saturation. In the present study, we used $\pm 31.25 \mathrm{kHz}$ of bandwidth and $1^{\circ}$ of flip angle with fewer sophisticated postprocessing steps to acquire ZTE skull MR imaging; these images were different from those used in previous studies. ${ }^{9,20,21,26}$ We initially aimed to evaluate the clinical diagnostic feasibility of ZTE skull MR imaging for detecting skull fracture, compared with CT; therefore, we focused on obtaining sufficient image quality with an optimal signal-tonoise ratio to interpret the fracture and suture lines in the skull on the visual analysis. With this perspective of visual qualitative analysis, we were not concerned about other structures such as the paranasal sinuses in the facial bones, which require a higher bandwidth for depicting in detail.

Several limitations of this study should be considered when interpreting the results. First, there was an unavoidable selection bias because the data from all patients were evaluated retrospectively, the sample size was small, and the study was conducted in a single center. Second, in this study, we evaluated only patients with head trauma; therefore, the representation of other skull pathologies is limited. This feature may have a different effect on the image quality for interpretation. Third, we did not use the sophisticated formula in the previous study ${ }^{26}$ to obtain the normalized bone tissue signal because we were focused on the morphologic perspective of ZTE skull MR imaging with simple and easy postprocessing. In the current study, it was sufficient to apply a simple noise threshold and a bias-correction algorithm to enhance bonetissue signal intensities and correct signal inhomogeneity due to coil geometry; therefore, complex scaling was not required. However, this approach to obtain the normalized bone tissue signal had an inherent limitation of value consistency because it was scaled to the background noise. A relatively low signal-to-noise ratio in ZTE skull MR imaging may indeed induce inhomogeneity of the signal intensity, which may then affect the results of the normalized bone tissue signal. Fourth, manual ROI selection, which was used for normalization, was reader-dependent and small; therefore, it may have affected the results, though we analyzed the interobserver reliability. Fifth, the ZTE sequence allows fast and quiet scanning to obtain skull images; however, it takes approximately 5 minutes, which allows the possibility of motion artifacts. We expect that further studies with larger sample sizes and various targeted patients will be conducted to validate our results in the near future.

\section{CONCLUSIONS}

In the current study, ZTE skull MR imaging generated a CT-like image with positive contrast for the skull by postprocessing, and it showed diagnostic image quality comparable with that of CT images for evaluating suture lines and traumatic skull lesions. It also showed a good correlation with CT images in skull-thickness measurement, and the normalized bone tissue signal was relatively consistent. Therefore, we believe that ZTE skull MR imaging may broaden the indications of MR imaging examinations, especially for radiosensitive patients with trauma such as pediatric patients or pregnant women because of its inherent benefit of not generating radiation. In addition, ZTE skull MR imaging may be helpful in MR imaging-dependent technologies such as PET/MR imaging reconstruction or MR imaging-guided radiation therapy and in procedures that provide useful information regarding attenuation correction or anatomic details.

\section{ACKNOWLEDGMENTS}

The authors would like to thank Michael Carl and Robert D. Peters at GE Healthcare for their advice regarding technical background of ZTE sequences and its postprocessing steps during the revision process. We also thank Elsevier Language Editing Service for the English language review and editing (http://webshop. elsevier.com/languageservices/languageediting/).

\section{REFERENCES}

1. Blystad I, Warntjes JB, Smedby O, et al. Synthetic MRI of the brain in a clinical setting. Acta Radiol 2012;53:1158-63 CrossRef Medline

2. Du J, Carl M, Bydder M, et al. Qualitative and quantitative ultrashort echo time (UTE) imaging of cortical bone. J Magn Reson 2010; 207:304-11 CrossRef Medline

3. Zeman RK, Fox SH, Silverman PM, et al. Helical (spiral) CT of the abdomen. AJR Am J Roentgenol 1993;160:719-25 CrossRef Medline

4. Kalender WA, Seissler W, Klotz E, et al. Spiral volumetric CT with single-breath-hold technique, continuous transport, and continuous scanner rotation. Radiology 1990;176:181-83 CrossRef Medline

5. Crawford CR, King KF. Computed tomography scanning with simultaneous patient translation. Med Phys 1990;17:967-82 CrossRef Medline

6. Vock P, Soucek M, Daepp M, et al. Lung: spiral volumetric CT with single-breath-hold technique. Radiology 1990;176:864-67 CrossRef Medline

7. Mettler FA Jr, Bhargavan M, Faulkner K, et al. Radiologic and nuclear medicine studies in the United States and worldwide: frequency, radiation dose, and comparison with other radiation sources-1950-2007. Radiology 2009;253:520-31 CrossRef Medline 8. Schauer DA, Linton OW. National Council on Radiation Protection 
and Measurements report shows substantial medical exposure increase. Radiology 2009;253:293-96 CrossRef Medline

9. Wiesinger F, Sacolick LI, Menini A, et al. Zero TE MR bone imaging in the head. Magn Reson Med 2016;75:107-14 CrossRef Medline

10. Robson MD, Gatehouse PD, Bydder M, et al. Magnetic resonance: an introduction to ultrashort TE (UTE) imaging. J Comput Assist Tomogr 2003;27:825-46 CrossRef Medline

11. Robson MD, Bydder GM. Clinical ultrashort echo time imaging of bone and other connective tissues. NMR Biomed 2006;19:765-80 CrossRef Medline

12. Du J, Bydder M, Takahashi AM, et al. Short T2 contrast with threedimensional ultrashort echo time imaging. Magn Reson Imaging 2011;29:470-82 CrossRef Medline

13. Madio DP, Lowe IJ. Ultra-fast imaging using low flip angles and FIDs. Magn Reson Med 1995;34:525-29 CrossRef Medline

14. Grodzki DM, Jakob PM, Heismann B. Ultrashort echo time imaging using pointwise encoding time reduction with radial acquisition (PETRA). Magn Reson Med 2012;67:510-18 CrossRef Medline

15. Idiyatullin D, Corum C, Park JY, et al. Fast and quiet MRI using a swept radiofrequency. J Magn Reson 2006;181:342-49 CrossRef Medline

16. Keereman V, Fierens Y, Broux T, et al. MRI-based attenuation correction for PET/MRI using ultrashort echo time sequences. $J$ Nucl Med 2010;51:812-88 CrossRef Medline

17. Catana $\mathrm{C}$, van der Kouwe A, Benner $\mathrm{T}$, et al. Toward implementing an MRI-based PET attenuation-correction method for neurologic studies on the MR-PET brain prototype. J Nucl Med 2010;51: 1431-38 CrossRef Medline
18. Johansson A, Karlsson M, Nyholm T. CT substitute derived from MRI sequences with ultrashort echo time. Med Phys 2011;38: 2708-14 CrossRef Medline

19. Delso G, Zeimpekis K, Carl M, et al. Cluster-based segmentation of dual-echo ultra-short echo time images for PET/MR bone localization. EJNMMI Phys 2014;1:1-13 CrossRef Medline

20. Delso G, Wiesinger F, Sacolick LI, et al. Clinical evaluation of zeroecho-time MR imaging for the segmentation of the skull. J Nucl Med 2015;56:417-22 CrossRef Medline

21. Breighner RE, Endo Y, Konin GP, et al. Technical developments: zero echo time imaging of the shoulder-enhanced osseous detail by using MR imaging. Radiology 2018;286:960-66 CrossRef Medline

22. Alibek S, Vogel M, Sun W, et al. Acoustic noise reduction in MRI using Silent Scan: an initial experience. Diagn Interv Radiol 2014;20: 360-63 CrossRef Medline

23. Landis JR, Koch GG. The measurement of observer agreement for categorical data. Biometrics 1977;33:159-74 CrossRef Medline

24. Johnson EM, Vyas U, Ghanouni P, et al. Improved cortical bone specificity in UTE MR imaging. Magn Reson Med 2017;77:684-95 CrossRef Medline

25. Grodzki DM, Jakob PM, Heismann B. Correcting slice selectivity in hard pulse sequences. J Magn Reson 2012;214:61-67 CrossRef Medline

26. Wiesinger F, Bylund M, Yang J, et al. Zero TE-based pseudo-CT image conversion in the head and its application in PET/MR attenuation correction and MR-guided radiation therapy planning. Magn Reson Med 2018;80:1440-51 CrossRef Medline 